\title{
Towards an Improvement of Fourier Transform
}

\author{
Khalid Aznag ${ }^{1}$, Toufik Datsi², Ahmed El Oirrak ${ }^{3}$, Essaid El Bachari ${ }^{4}$ \\ Computer Systems Engineering Laboratory \\ Faculty of Sciences Semlalia \\ Marrakech, Morocco
}

\begin{abstract}
With the development of information technology and the coming period of large data, the image signals play an increasingly more significant role in our life because of the phenomenal development of system correspondence innovation, and the comparing high proficiency image handling strategies are requested earnestly. The Fourier transform is an important image processing tool, which is used in a wide range of applications, such as image filtering, image analysis, image compression and image reconstruction. It 's the simplest among the other transformation method used in mathematics. The real time consumption is lesser due to this method. It has a vast use in image processing, particularly object 2D, 3D and other representation. This paper proposes a new Fourier transform which is called Non Uniform Fourier Transform (NUFT). The proposed descriptor takes into consideration the change of point index. Also, an application is made on 2D set of points and a real image. The main advantages of the proposed transform are invariance under change of index point and robustness to noise. Also, the extraction of invariant under rotation and affinity is immediate because the linearity is assured. The proposed descriptor is tested on MPEG 7 database and compared with the normal Fourier transform to shows its efficiency. The experimental results prove the effectiveness of the proposed descriptor.
\end{abstract}

Keywords—Fourier transform; NUFT; noise; invariant

\section{INTRODUCTION}

Fourier transform is an interesting image processing tool which used to decompose an image into sine and cosine components. Fourier transform is used in several fields such as image processing and filters, transformation, representation, etc. Historically, one of the most widely used shape description methods is Fourier descriptors (FD) [1], [2], [3], [4]. The discrete Fourier transform (DFT) is one of the most fundamental and important numerical algorithms which plays a central role in the image processing area, including image denoising [5], image feature extraction [6], and compressed sensing [7]. The Fast Fourier Transform (FFT) [8] which computes the DFT of an n-size signal in $\mathrm{O}(\mathrm{n} \log \mathrm{n})$ time greatly simplifies the complexity of DFT and gets a wide range of applications. In [9], [10] and [11], [12] are implementations of the discrete Fourier transform (DFT). Fourier transform hypotheses of central importance in a vast range of applications in engineering, applied mathematics, and physical science. In addition, Fourier transform is a mathematical concept which suited extremely well for signal analysis. In [13], [14] authors proposed sparse fast Fourier transform for one-dimension (1D-SFFT) signal which is faster than traditional DFT. However, the two-dimensional image signal is more broadly used, and a two-dimensional sparse
Fourier transform cannot simply be constructed with a onedimensional sparse Fourier transform. Sheng Shi et al in [15] proposed a new fast two-dimensional Fourier transform (2DSFFT) that takes advantage of image sparsity.

In this work, the advantage of our method is that it is systematic and it allows obtaining the analytical form of all invariant polynomials of a given order, which was not the case using the Suck method and Flusser [16].

The rest of this paper will be organized as follows: Section II provides an overview of related work. Section III describes normal Fourier descriptors and the proposed Fourier transform. In Section IV the experiments are presented. The results and discussions are presented in Section V. Section VI contains the conclusion and the future work highlighted in Section VII.

\section{RELATED WORKS}

Fourier transform is one of the oldest and well-known methods in the field of the mathematics. It used in a wide range of applications, such as image filtering, image analysis, image reconstruction, and image compression. In the literature, several papers have described methods for approximating one-dimensional Non uniform Fast Fourier Transforms by interpolating an oversampled Fast Fourier transforms, start with [17] and including [18], [19], [20], [21], [22], [23], [24], [25], [26], [27]. In [28] the fundamentals of Fourier transform, Fourier series, discrete Fourier transform and fast Fourier transform with simple examples and review of Fourier transform to supply a clear understanding of its applications in power quality issues. In [29], the author presents a novel method for improving the Fast Fourier Transform (FFT) based spectral estimation for the diagnosis of faults in induction motors. In [30], the authors propose a new method for optical image encryption using fractional Fourier transform (FRFT). Two-dimensional fast Fourier transform (2D-FFT) is successfully applied to analyse images [31]. Image compression technique that uses real Fourier transform is proposed by Kekre et al in [32]. Their technique is applied on the image in three ways: Row transform, Column transform, and Full transform. Aznag et al. in [33] applied this new descriptor for 3D parameterized point set and 3D curve. Previous research has demonstrated that there is a nonuniform Fourier transform [16], [34], but the problem treated in this new work is not the spacing between samples, but simply the change in order of points in storage or manipulation of those points. 


\section{METHODOLOGY}

This section discusses the proposed descriptor. We first give an overview of the normal Fourier descriptor. Then, we describe more in detail our proposed Fourier descriptor.

\section{A. Normal Fourier Descriptor}

1) Fourier descriptor for two-dimensional indexed point set: To define Fourier descriptor (FD) for 2D indexed point set; Let $X(x(n), y(n)) n=1 \ldots N$, denotes a closed contour with $\mathrm{N}$ is the number of points on the normalized contour and $2 \pi$ as length, then the Fourier descriptors are given by equations. (1), (2) and (3).

$Z(k)=\left(\begin{array}{l}u(k) \\ v(k)\end{array}\right)$

With

$u(k)=\frac{1}{N} \sum_{n=1}^{N} x(n) e^{-j \frac{2 \pi k n}{N}}$

$v(k)=\frac{1}{N} \sum_{n=1}^{N} y(n) e^{-j \frac{2 \pi k n}{N}}$

For $\mathrm{k}=1 \ldots \mathrm{N}$

Where $\mathrm{u}$ and $\mathrm{v}$ represent the Fourier descriptors of $\mathrm{x}$ and $\mathrm{y}$ respectively.

2) Shift theorem and transformation effect: Let $X$ and $\tilde{X}$ are two objects having the same shape but with a shift in starting points, then (Equations. (4), (5) and (6)):

$\tilde{Z}(k)=e^{j k l_{0}} A Z(k)+B \delta_{k}$

For all integer $\mathrm{k}$,

$Z(k)=(u(k), v(k))$

and

$\tilde{Z}(k)=(\tilde{u}(k), \tilde{v}(k))$

Are respectively the bi-dimensional Fourier descriptor vectors of $X$ and $\tilde{X}$.

Where

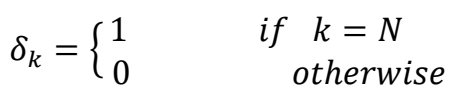

Is the Kronecker symbol (Equation. (7)). The real $l_{0}$ denotes the difference between starting points on a contour and its transformed.

\section{B. Proposed Fourier Descriptor}

In this section, we present the proposed descriptor. As normal Fourier transform is dependent on point index. So, to solve this problem we define the novel descriptor. We apply the proposed Fourier transform for the 2D parameterized point set and the binary objects. The idea of our descriptor is simple and easy, we replace integer $\mathrm{k}$ by parameter $\tau$ (see Equation. (16)).

In general, we have two cases:
- A structured set of points, indexed by $\mathrm{n}$ integer which represents the order of points.

- Unstructured set of points, in this case the order is not respected and we propose the use of another characteristic of a point, which is independent of the order.

3) Novel Fourier descriptor for two-dimensional indexed point set: To define novel Fourier descriptor (FD) for 2D indexed point set; Let $X(\boldsymbol{\tau})=(\mathbf{x}(\boldsymbol{\tau}), \mathbf{y}(\boldsymbol{\tau}))$ denotes a $2 \mathrm{D}$ set of point having $\boldsymbol{\tau}$ as parameter and $\mathrm{N}$ is the number of points, then the novel 2D Fourier descriptors is defined by.

$\left\{\begin{array}{l}u(k)=\frac{1}{N} \sum_{\tau} x(\tau) e^{-j \frac{k \tau}{T}} \\ v(k)=\frac{1}{N} \sum_{\tau} y(\tau) e^{-j \frac{k \tau}{T}}\end{array}\right.$

Where

$T=\int \tau d \tau$

4) Linearity: Let $X=(x(\tau), y(\tau))$ and $\tilde{X}=(\tilde{x}(\tau), \tilde{y}(\tau))$ denote two shapes having the same shape with an affine transform difference i.e (Equations. (9), (10), (11), (12), (13) and (14)):

$\tilde{X}=A X$

Where

$A=\left(\begin{array}{l}a_{11} a_{12} \\ a_{21} a_{22}\end{array}\right)$

So

$\tilde{x}=a_{11} x(\tau)+a_{12} y(\tau)$

$\tilde{y}=a_{21} x(\tau)+a_{22} y(\tau)$

Then

$\tilde{u}(\tau)=\frac{1}{N} \sum_{n=1}^{N} \tilde{x}(\tau) e^{-\frac{j k \tau}{T}}$

$=\frac{1}{N} \sum_{n=1}^{N}\left(a_{11} x(\tau)+a_{12} y(\tau)\right) e^{-\frac{j k \tau}{T}}$

$=a_{11} u(k)+a_{12} v(k)$

The same for:

$\tilde{v}(k)=a_{21} u(k)+a_{22} v(k)$

In case of rotation transformation, a $2 \times 2$ transformation matrix is defined by

$\mathrm{A}=\left(\begin{array}{cc}\cos \theta & -\sin \theta \\ \sin \theta & \cos \theta\end{array}\right)$

Where $\theta$ is the angle of rotation. This principle of linearity allows us to extract invariant easily from shapes $X$ and $\tilde{X}$. 


\section{EXPERIMENTS}

\section{A. Application on Sets of Points}

In these experiments, we have four sets of $2 \mathrm{D}$ points with $x$ and $y$ coordinates shown in Fig. 1. We present the experimental test of our proposed descriptor in four shapes that are shown in Fig. 1(a), 1(b), 1(c) and 1(d). Using Equation (16), the parameter $\tau$ used in this experiment is defined by

$\tau=\sqrt{x^{2}+y^{2}}$

\section{B. Application on Real Image}

To test our approach; we apply our method on the MPEG7 database [35]. In this database there are 70 classes of shapes, each one has 20 members as shown in Fig. 6. In this section we applied our approach to a real image, an MPEG7 image of size $750 \times 531$ is presented in Fig. 2. To obtain contour points we can browse edge image using row by row or column by column. So, the coordinates of contours have not the same index using previous browses. Starting points on each contour are presented by a circle (as shown in Fig. 3). Not only the starting points are different but also the order of other points. Fig. 4 shows results with contours of an elephant.

\section{Noise Effect}

In order to test our new Fourier transform for noise, we add noise to the Elephant image (see Fig. 5). The percentage of added noise is $10 \%$ and we take the first 10 coefficients (development into Novel transformed of coordinates $\mathrm{x}$ ).

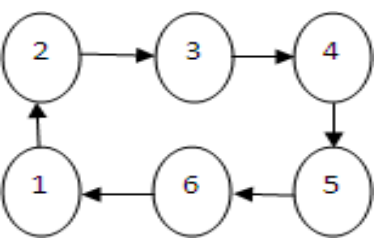

(a) The First Shape.

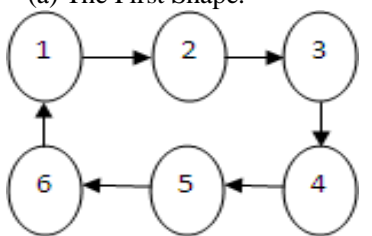

(c) The Third Shape.

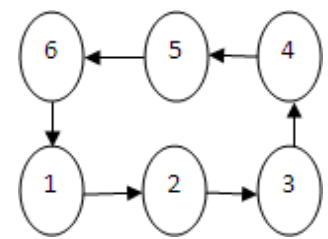

(b) The Second Shape.

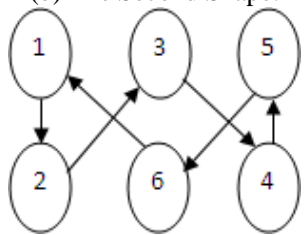

(d) The Fourth Shape.
Fig. 1. A Set of Points with Associated 2D Shapes.

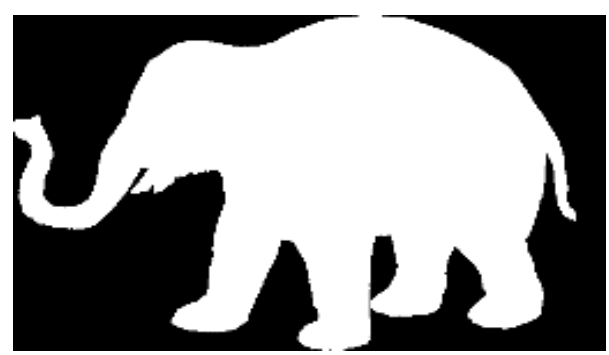

Fig. 2. Elephant Image.

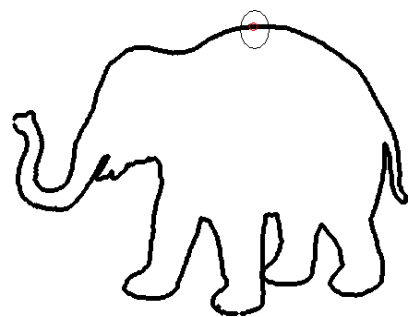

(a) Elephant contour and a starting point.

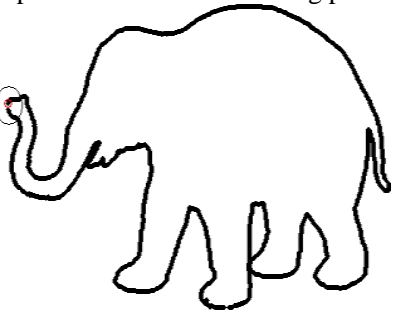

(b) Elephant contour with another starting point.

Fig. 3. The Contours of Elephant Represent the Same Content but the Order of Points is not the Same.

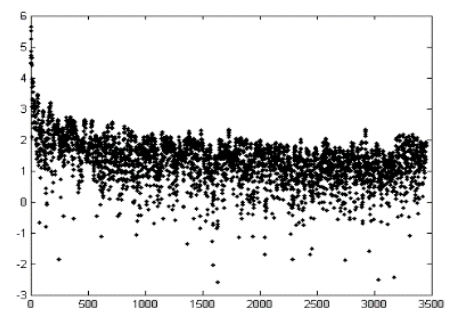

(a) Graph of abs $|\log (\mathrm{U})|$ for Contour 3.a)

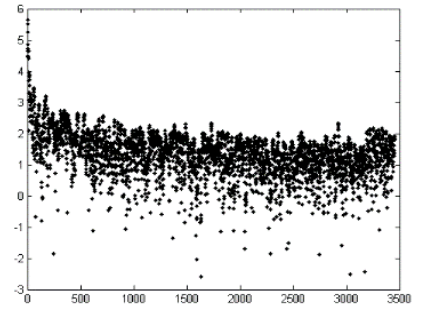

(b) Graph of abs $|\log (\mathrm{U})|$ for Contour 3.b)

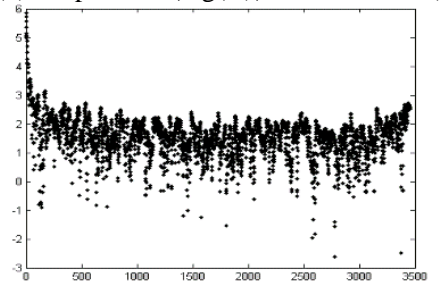

(c) Graph of $\operatorname{abs}|\log (\mathrm{V})|$ for Contour 3.a)

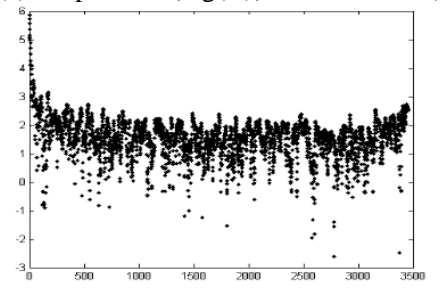

(d) Graph of $a b s|\log (\mathrm{V})|$ for Contour 3.b)

Fig. 4. Graphs Represent Abs $|\log (\mathrm{U})|$ (a) and (b), Graphs Represent Abs $|\log (\mathrm{V})|(\mathrm{c})$ and (d). 


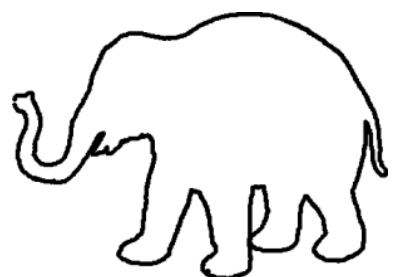

(a) Original elephant image.

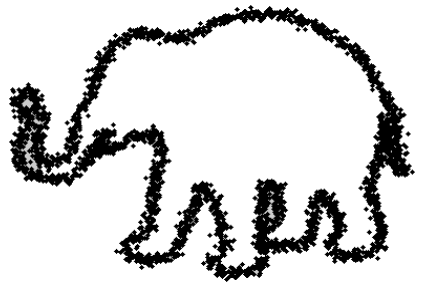

(b) Noisy elephant image.
Fig. 5. Original and Noisy Elephant Images.

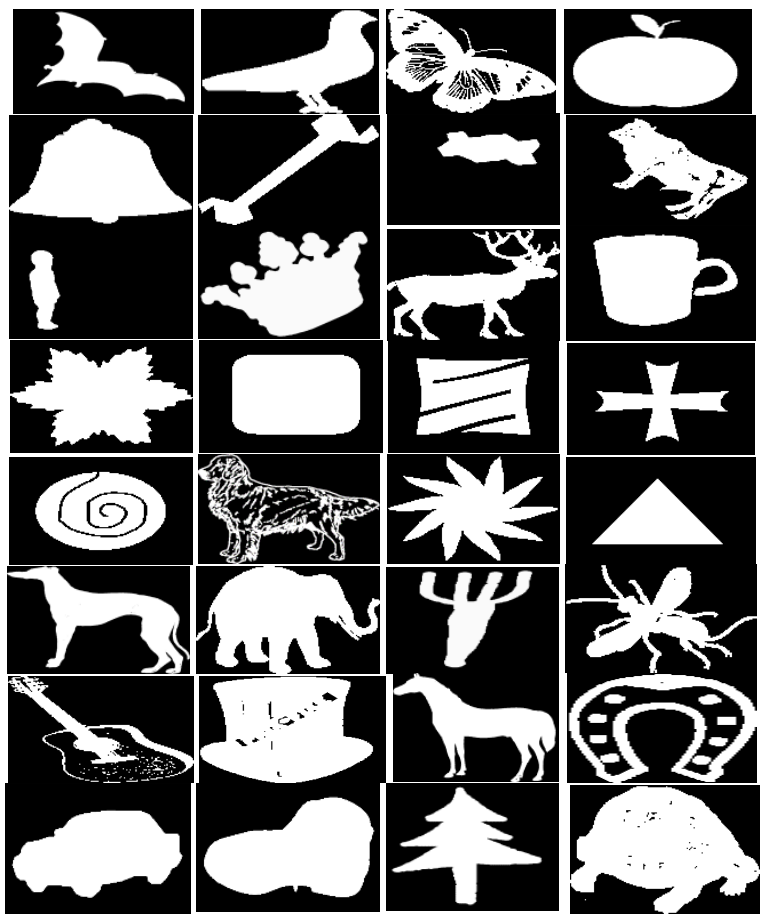

Fig. 6. Example of MPEG7 Database.

\section{RESUlTS AND DISCUSSION}

The problem treated in this work is not the spacing between samples, but simply the change in the order of points in storage or manipulation of those points. We notice that the Normal Fourier transform requires $O(N \log N)$ to compute $\mathrm{N}$ Fourier modes from $\mathrm{N}$ data points. Also, novel transform achieves the same $O(N \log N)$ computational complexity.

Tables I, II, III and IV we present the $\mathrm{x}$ and $\mathrm{y}$ coordinates of the points with different indices of the four shapes given in Fig. 1. The polor radius for each point is shown in Table V. In Tables VI, VII, VIII, and IX we present the normal Fourier transform for each shape given in Fig. 1, we can show no equality of coefficients. In Tables X, XI, XII, and XIII we present the novel Fourier transform for each shape. We see that the novel two components $\mathrm{U}$ and $\mathrm{V}$ are the same. It's clear from Tables VI, VII, VIII, IX, X, XI, XII, and XIII which the proposed Fourier transform is efficient.

TABLE. I. COORDINATES X AND Y FOR SHAPE (FIG.1.A)

\begin{tabular}{|l|l|l|l|l|l|l|}
\hline Index & 1 & 2 & 3 & 4 & 5 & 6 \\
\hline Coordinates $\mathbf{x}$ & 1 & 1 & 1.5 & 2 & 2 & 1.5 \\
\hline Coordinates $\mathbf{y}$ & 1 & 2 & 2 & 2 & 1 & 1 \\
\hline
\end{tabular}

TABLE. II. COORDINATES X AND Y FOR SHAPE (FIG.1.B)

\begin{tabular}{|l|l|l|l|l|l|l|}
\hline Index & 1 & 2 & 3 & 4 & 5 & 6 \\
\hline Coordinates x & 1 & 1.5 & 2 & 2 & 1.5 & 1 \\
\hline Coordinates y & 1 & 1 & 1 & 2 & 2 & 2 \\
\hline
\end{tabular}

TABLE. III. COORDINATES X AND Y FOR SHAPE (FIG.1.C)

\begin{tabular}{|l|l|l|l|l|l|l|}
\hline Index & 1 & 2 & 3 & 4 & 5 & 6 \\
\hline Coordinates x & 1 & 1.5 & 2 & 2 & 1.5 & 1 \\
\hline Coordinates y & 2 & 2 & 2 & 1 & 1 & 1 \\
\hline
\end{tabular}

TABLE. IV. COORDINATES X AND Y FOR SHAPE (FIG.1.D)

\begin{tabular}{|l|l|l|l|l|l|l|}
\hline Index & 1 & 2 & 3 & 4 & 5 & 6 \\
\hline Coordinates x & 1 & 1 & 1.5 & 2 & 2 & 1.5 \\
\hline Coordinates y & 2 & 1 & 2 & 1 & 2 & 1 \\
\hline
\end{tabular}

TABLE. V. RAdious of Shapes (Fig.1.A), (Fig.1.B), (FIg.1.C) AND (FIG.1.D)

\begin{tabular}{|l|l|l|l|l|l|l|}
\hline$\tau$ for shape 1.a) & 1.41 & 2.23 & 2.50 & 2.82 & 2.23 & 1.80 \\
\hline$\tau$ for shape 1.b) & 1.41 & 1.80 & 2.23 & 2.82 & 2.50 & 2.23 \\
\hline$\tau$ for shape 1.c) & 2.23 & 2.50 & 2.82 & 2.23 & 1.80 & 1.41 \\
\hline $\boldsymbol{\tau}$ for shape 1.d) & 2.23 & 1.41 & 2.23 & 2.23 & 2.82 & 1.80 \\
\hline
\end{tabular}

TABLE. VI. NORMAL Fourier TRANSForm OF SHAPE (Fig.1.A)

\begin{tabular}{|l|l|}
\hline $\mathbf{U}$ & $\mathbf{V}$ \\
\hline$-0.0000+0.2887 \mathrm{i}$ & $-0.3333-0.0000 \mathrm{i}$ \\
\hline $0.0000+0.0000 \mathrm{i}$ & $0.0000-0.0000 \mathrm{i}$ \\
\hline $0-0.0000 \mathrm{i}$ & $0.1667-0.0000 \mathrm{i}$ \\
\hline$-0.0000-0.0000 \mathrm{i}$ & $-0.0000-0.0000 \mathrm{i}$ \\
\hline$-0.0000-0.2887 \mathrm{i}$ & $-0.3333-0.0000 \mathrm{i}$ \\
\hline $1.5000+0.0000 \mathrm{i}$ & $1.5000+0.0000 \mathrm{i}$ \\
\hline
\end{tabular}

TABLE. VII. NORMAL FOURIER TRANSFORM OF SHAPE (FIG.1.B)

\begin{tabular}{|l|l|}
\hline $\mathbf{U}$ & $\mathbf{V}$ \\
\hline$-0.2500+0.1443 \mathrm{i}$ & $0.1667+0.2887 \mathrm{i}$ \\
\hline $0.0000-0.0000 \mathrm{i}$ & $0.0000-0.0000 \mathrm{i}$ \\
\hline $0.0000-0.0000 \mathrm{i}$ & $0.1667-0.0000 \mathrm{i}$ \\
\hline$-0.0000-0.0000 \mathrm{i}$ & $-0.0000-0.0000 \mathrm{i}$ \\
\hline$-0.2500-0.1443 \mathrm{i}$ & $0.1667-0.2887 \mathrm{i}$ \\
\hline $1.5000+0.0000 \mathrm{i}$ & $1.5000+0.0000 \mathrm{i}$ \\
\hline
\end{tabular}

TABLE. VIII. NORMAL FoURIER TRANSFORM OF SHAPE (FIG.1.C)

\begin{tabular}{|l|l|}
\hline $\mathbf{U}$ & $\mathbf{V}$ \\
\hline$-0.2500+0.1443 \mathrm{i}$ & $-0.1667-0.2887 \mathrm{i}$ \\
\hline $0.0000-0.0000 \mathrm{i}$ & $0.0000+0.0000 \mathrm{i}$ \\
\hline $0.0000-0.0000 \mathrm{i}$ & $-0.1667-0.0000 \mathrm{i}$ \\
\hline$-0.0000-0.0000 \mathrm{i}$ & $-0.0000-0.0000 \mathrm{i}$ \\
\hline$-0.2500-0.1443 \mathrm{i}$ & $-0.1667+0.2887 \mathrm{i}$ \\
\hline $1.5000+0.0000 \mathrm{i}$ & $1.5000+0.0000 \mathrm{i}$ \\
\hline
\end{tabular}


TABLE. IX. NORMAL FourIER TRANSFORM OF SHAPE (FIG.1.D)

\begin{tabular}{|l|l|}
\hline $\boldsymbol{U}$ & $\boldsymbol{V}$ \\
\hline$-0.0000+0.2887 \mathrm{i}$ & $0.0000-0.0000 \mathrm{i}$ \\
\hline $0.0000+0.0000 \mathrm{i}$ & $0.0000+0.0000 \mathrm{i}$ \\
\hline $0-0.0000 \mathrm{i}$ & $-0.5000-0.0000 \mathrm{i}$ \\
\hline$-0.0000-0.0000 \mathrm{i}$ & $-0.0000+0.0000 \mathrm{i}$ \\
\hline$-0.0000-0.2887 \mathrm{i}$ & $-0.0000-0.0000 \mathrm{i}$ \\
\hline $1.5000+0.0000 \mathrm{i}$ & $1.5000+0.0000 \mathrm{i}$ \\
\hline
\end{tabular}

TABLE. X. NOVEl Fourier TRANSFORM OF SHAPE (Fig.1.A)

\begin{tabular}{|l|l|}
\hline $\mathbf{U}$ & $\mathbf{V}$ \\
\hline$-0.9558-0.9468 \mathrm{i}$ & $-1.0020-0.9093 \mathrm{i}$ \\
\hline $0.0571+0.9518 \mathrm{i}$ & $0.1659+0.9746 \mathrm{i}$ \\
\hline $0.2418-0.4530 \mathrm{i}$ & $0.1739-0.5847 \mathrm{i}$ \\
\hline$-0.1428+0.2268 \mathrm{i}$ & $-0.2092+0.3785 \mathrm{i}$ \\
\hline $0.1581-0.1539 \mathrm{i}$ & $0.3136-0.1968 \mathrm{i}$ \\
\hline$-0.1102-0.0536 \mathrm{i}$ & $-0.2207-0.1324 \mathrm{i}$ \\
\hline
\end{tabular}

TABLE. XI. NOVEl Fourier TRANSForm OF SHAPE (FIG.1.B)

\begin{tabular}{|l|l|}
\hline $\mathbf{U}$ & $\mathbf{V}$ \\
\hline$-0.9558-0.9468 \mathrm{i}$ & $-1.0020-0.9093 \mathrm{i}$ \\
\hline $0.0571+0.9518 \mathrm{i}$ & $0.1659+0.9746 \mathrm{i}$ \\
\hline $0.2418-0.4530 \mathrm{i}$ & $0.1739-0.5847 \mathrm{i}$ \\
\hline$-0.1428+0.2268 \mathrm{i}$ & $-0.2092+0.3785 \mathrm{i}$ \\
\hline $0.1581-0.1539 \mathrm{i}$ & $0.3136-0.1968 \mathrm{i}$ \\
\hline$-0.1102-0.0536 \mathrm{i}$ & $-0.2207-0.1324 \mathrm{i}$ \\
\hline
\end{tabular}

TABLE. XII. NOVEl Fourier TRANSFORM OF ShaPe (FIG.1.C)

\begin{tabular}{|l|l|}
\hline $\mathbf{U}$ & $\mathbf{V}$ \\
\hline$-0.9558-0.9468 \mathrm{i}$ & $-1.0020-0.9093 \mathrm{i}$ \\
\hline $0.0571+0.9518 \mathrm{i}$ & $0.1659+0.9746 \mathrm{i}$ \\
\hline $0.2418-0.4530 \mathrm{i}$ & $0.1739-0.5847 \mathrm{i}$ \\
\hline$-0.1428+0.2268 \mathrm{i}$ & $-0.2092+0.3785 \mathrm{i}$ \\
\hline $0.1581-0.1539 \mathrm{i}$ & $0.3136-0.1968 \mathrm{i}$ \\
\hline$-0.1102-0.0536 \mathrm{i}$ & $-0.2207-0.1324 \mathrm{i}$ \\
\hline
\end{tabular}

TABLE. XIII. NOVEl Fourier TRANSFORM OF SHAPE (FIG.1.D)

\begin{tabular}{|l|l|}
\hline $\mathbf{U}$ & $\mathbf{V}$ \\
\hline$-0.9558-0.9468 \mathrm{i}$ & $-1.0020-0.9093 \mathrm{i}$ \\
\hline $0.0571+0.9518 \mathrm{i}$ & $0.1659+0.9746 \mathrm{i}$ \\
\hline $0.2418-0.4530 \mathrm{i}$ & $0.1739-0.5847 \mathrm{i}$ \\
\hline$-0.1428+0.2268 \mathrm{i}$ & $-0.2092+0.3785 \mathrm{i}$ \\
\hline $0.1581-0.1539 \mathrm{i}$ & $0.3136-0.1968 \mathrm{i}$ \\
\hline$-0.1102-0.0536 \mathrm{i}$ & $-0.2207-0.1324 \mathrm{i}$ \\
\hline
\end{tabular}

In Tables XIV and XV, we see that coefficients $\mathrm{U}$ and $\mathrm{V}$ are the same for two contours, only the first 10 coefficients using novel Fourier transform are presented. Graphic representation of $a b s|\log (U)|$ and $a b s|\log (V)|$ for all coefficients is given in Fig. 4. We note that coefficients are always in the same order of magnitude (see Table XVI).
TABLE. XIV. NOVEL FOURIER TRNASFORM OF COORDINATES X FOR TwO CONTOURS 4(A) AND 4(B)

\begin{tabular}{|l|l|}
\hline $\mathbf{U}$ & $\mathbf{U}$ \\
\hline $1.5477-2.3974 \mathrm{i}$ & $1.5477-2.3974 \mathrm{i}$ \\
\hline$-1.0556-2.2168 \mathrm{i}$ & $-1.0556-2.2168 \mathrm{i}$ \\
\hline$-1.8839-0.1026 \mathrm{i}$ & $-1.8839-0.1026 \mathrm{i}$ \\
\hline$-0.5257+1.1775 \mathrm{i}$ & $-0.5257+1.1775 \mathrm{i}$ \\
\hline $0.7773+0.4266 \mathrm{i}$ & $0.7773+0.4266 \mathrm{i}$ \\
\hline $0.3836-0.7878 \mathrm{i}$ & $0.3836-0.7878 \mathrm{i}$ \\
\hline$-0.8005-0.6661 \mathrm{i}$ & $-0.8005-0.6661 \mathrm{i}$ \\
\hline$-1.0159+0.4648 \mathrm{i}$ & $-1.0159+0.4648 \mathrm{i}$ \\
\hline$-0.1105+1.0289 \mathrm{i}$ & $-0.1105+1.0289 \mathrm{i}$ \\
\hline $0.6326+0.5173 \mathrm{i}$ & $0.6326+0.5173 \mathrm{i}$ \\
\hline
\end{tabular}

TABLE. XV. NOVEL FOURIER TRANSFORM OF COORDINATES Y FOR TwO CONTOURS 4(A) AND 4(B)

\begin{tabular}{|l|l|}
\hline $\mathbf{V}$ & $\mathbf{V}$ \\
\hline $1.5950-3.0604 \mathrm{i}$ & $1.5950-3.0604 \mathrm{i}$ \\
\hline$-1.8277-2.4957 \mathrm{i}$ & $-1.8277-2.4957 \mathrm{i}$ \\
\hline$-2.5307+0.5763 \mathrm{i}$ & $-2.5307+0.5763 \mathrm{i}$ \\
\hline$-0.2078+2.0807 \mathrm{i}$ & $-0.2078+2.0807 \mathrm{i}$ \\
\hline $1.6373+0.5444 \mathrm{i}$ & $1.6373+0.5444 \mathrm{i}$ \\
\hline $0.7498-1.3746 \mathrm{i}$ & $0.7498-1.3746 \mathrm{i}$ \\
\hline$-1.1345-1.0216 \mathrm{i}$ & $-1.1345-1.0216 \mathrm{i}$ \\
\hline$-1.2702+0.7405 \mathrm{i}$ & $-1.2702+0.7405 \mathrm{i}$ \\
\hline $0.2403+1.3063 \mathrm{i}$ & $0.2403+1.3063 \mathrm{i}$ \\
\hline $1.0990+0.1632 \mathrm{i}$ & $1.0990+0.1632 \mathrm{i}$ \\
\hline
\end{tabular}

TABLE. XVI. RESULT $a b s|\log (U)|_{\text {FOR FIG. 5(A) AND }} a b s|\log (U)|_{\text {FOR }}$ FIG. 5(B)

\begin{tabular}{|l|l|}
\hline $\mathbf{U}$ & $\mathbf{U}$ \\
\hline 5.6537 & 5.6538 \\
\hline 5.5034 & 5.5035 \\
\hline 5.2400 & 5.2399 \\
\hline 4.8595 & 4.8591 \\
\hline 4.4849 & 4.4839 \\
\hline 4.4731 & 4.4717 \\
\hline 4.6457 & 4.6445 \\
\hline 4.7160 & 4.7150 \\
\hline 4.6394 & 4.6388 \\
\hline 4.4033 & 4.4035 \\
\hline
\end{tabular}

\section{CONCLUSION}

This paper presents a new Fourier transform to solve the problem of index of a point. The radius parameter is used in the development. The experimental results show that NUFT presents substantial advantages than normal Fourier transform. Shift theorem is available only where the Shift is linear, but when the order of point is randomly this theorem is not valid. The advantage of our transform is invariance under change of index point, and especially robustness to noise. 


\section{FUTURE WORK}

In the future, the authors are interested to implement our descriptor on speech signal and for 3D objects (mesh, surfaces) and using neural network.

\section{REFERENCES}

[1] A. El-ghazal, O. Basir, and S. Belkasim, "Invariant curvature-based Fourier shape descriptors," Journal of Visual Communication and Image Representation, 23(4), pp.622-633, 2012.

[2] Y. Xu, Z. Xie, Z. Chen, and L. Wu, "Shape similarity measurement model for holed polygons based on position graphs and Fourier descriptors," International Journal of Geographical Information Science, 31(2), pp. 253-279, 2017.

[3] S.D. Choudhury, and T. Tjahjadi, "Silhouette-based gait recognition using Procrustes shape analysis and elliptic Fourier descriptors," Pattern Recognition, 45(9), pp.3414-3426, 2012.

[4] C.Y. Wang, and Y. Zhang, "Affine invariant feature extraction algorithm based on multiscale autoconvolution combining with texture structure analysis," Optik-International Journal for Light and Electron Optics, 125(13), pp. 3184-3189, 2013.

[5] P. Luo, X. Qu, X. Qing, and J. Gu, "CT Image Denoising Using Double Density Dual Tree Complex Wavelet with Modified Thresholding," In 2018 2nd International Conference on Data Science and Business Analytics (ICDSBA), pp. 287-290, 2018.

[6] W. Zhao, and S. Du, "Learning multiscale and deep representations for classifying remotely sensed imagery," ISPRS Journal of Photogrammetry and Remote Sensing, 113, pp. 155-165, 2016.

[7] S. Shi, R. Xiong, S. Ma, X. Fan, and W. Gao, "Image compressive sensing using overlapped block projection and reconstruction," In 2015 IEEE International Symposium on Circuits and Systems (ISCAS), pp.1670-1673, 2015.

[8] D. Li, X. Zhang, and D. Tu, “ An FFT-Based Technique for Underwater Image Stitching," In International Workshop of Advanced Manufacturing and Automation, pp. 527-535, 2018.

[9] D. Ravi, C. Wong, B. Lo, and G.Z. Yang, "Deep learning for human activity recognition: A resource efficient implementation on low-power devices, "In 2016 IEEE 13th International Conference on Wearable and Implantable Body Sensor Networks (BSN), pp. 71-76, 2016.

[10] A. Abdalla, and C. Green, "Utilisation of Fast Fourier Transform and Least-squares Modification of Stokes formula to compile a gravimetric geoid model over Khartoum State: Sudan," Arabian Journal of Geosciences, 9(3), 236, 2016.

[11] Z. Li, H. Jia, Y. Zhang, T. Chen, L. Yuan, Cao L., and X. Wang, "AutoFFT: a template-based FFT codes auto-generation framework for ARM and X86 CPUs," In Proceedings of the International Conference for High Performance Computing, Networking, Storage and Analysis, p. $25,2019$.

[12] D. Takahashi, "Parallel FFT Algorithms for Shared-Memory Parallel Computers," In Fast Fourier Transform Algorithms for Parallel Computers, pp. 69-76, 2019.

[13] H. Hassanieh, P. Indyk, D. Katabi, and E. Price, "Simple and practical algorithm for sparse Fourier transform. In Proceedings of the twentythird annual ACM-SIAM symposium on Discrete Algorithms," Society for Industrial and Applied Mathematics, pp.1183-1194, 2012.

[14] A. Pfeifer, and V. Lohweg, "Detection of commercial offset printing using an adaptive software architecture for the DFT," In 2016 IEEE 21st International Conference on Emerging Technologies and Factory Automation (ETFA), pp. 1-4, 2016.

[15] S. Shi, R. Yang, and H. You, "A new two-dimensional Fourier transform algorithm based on image sparsity," IEEE International Conference on Acoustics, Speech and Signal Processing (ICASSP), pp. 1373-1377, 2017.

[16] H. Li, X. Jin, N. Yang, and Z. Yang, "The recognition of landed aircrafts based on PCNN model and affine moment invariants," Pattern Recognition Letters, 51, pp. 23-29, 2015.
[17] G., Plonka, D., Potts, G., Steidl, and M.Tasche, "Fast Fourier Transforms for Nonequispaced Data," In Numerical Fourier Analysis, pp. 377-419, 2018.

[18] S.F., Potter, N.A., Gumerov, and R. Duraiswami, "Fast interpolation of bandlimited functions," In 2017 IEEE International Conference on Acoustics, Speech and Signal Processing (ICASSP), pp. 4516-4520, 2017.

[19] J. M. Lin, "Python Non-Uniform Fast Fourier Transform (PyNUFFT): multi-dimensional non-Cartesian image reconstruction package for heterogeneous platforms and applications to MRI," arXiv preprint arXiv:1710.03197, 2017.

[20] R. Matthysen, and D. Huybrechs, "Function approximation on arbitrary domains using Fourier extension frames," SIAM Journal on Numerical Analysis, 56(3), pp. 1360-1385, 2018.

[21] J. S. Walker, "Fast fourier transforms, " CRC press, 2017.

[22] G. Plonka, D. Potts, G. Steidl, and M. Tasche, "Fast Fourier Transforms for Nonequispaced Data," In Numerical Fourier Analysis, pp. 377-419, 2018.

[23] J. Schmid, T. Glatz, B. Zabihian, M Liu, W. Drexler, and O. Scherzer, "Nonequispaced grid sampling in photoacoustics with a nonuniform fast Fourier transform," Journal of biomedical optics, 21(1), 015005, 2016.

[24] L. Le Magoarou, and R. Gribonval, “ Are there approximate fast fourier transforms on graphs," In 2016 IEEE International Conference on Acoustics, Speech and Signal Processing (ICASSP), pp. 4811-4815, 2016.

[25] D. Ruiz-Antolin, and A. Townsend, "A nonuniform fast Fourier transform based on low rank approximation," SIAM Journal on Scientific Computing, 40(1), pp. A529-A547, 2018.

[26] T. Ou, "gNUFFTW: Auto-Tuning for High-Performance GPUAccelerated Non-Uniform Fast Fourier Transforms, " 2017.

[27] D. Potts, G. Steidl, and M. Tasche, "Fast Fourier transforms for nonequispaced data: A tutorial, " InModern Sampling Theory: Mathematics and Application, Birkhäuser, Boston, MA,pp.247-270, 2001.

[28] L. Saribulut, T.E.K.E. Ahmet, and M. Tumay, "Fundamentals and literature review of Fourier transform in power quality issues," Journal of Electrical and Electronics Engineering Research, 5(1), pp. 9-22, 2013.

[29] de Jesus Romero-Troncoso, R. "Multirate signal processing to improve FFT-based analysis for detecting faults in induction motors, " IEEE Transactions on industrial informatics, 13(3), pp. 1291-1300, 2016.

[30] M.B. Farah, R. Guesmi, A. Kachouri, and M. Samet, "A novel chaos based optical image encryption using fractional Fourier transform and DNA sequence operation," Optics \& Laser Technology, 121, 105777, 2020.

[31] Zhu, J., Balieu, R., Lu, X., and Kringos, N. (2018). "Microstructure evaluation of polymer-modified bitumen by image analysis using twodimensional fast Fourier transform, "Materials \& design, 137, 164-175.

[32] H.B. Kekre , T. Sarode, P. Natu, "ImageCompression Using Real Fourier Transform, Its Wavelet Transform And Hybrid Wavelet With DCT," International Journal of Advanced Computer Science and Applications, 4(5), 2013.

[33] K. Aznag, N. Kane, A. El oirrak, and E. El bachari, "New approach for 3D object recognition using non uniform fourier and moments coefficients," Journal of Engineering Science and Technology, 12(5), pp.1354-1365, 2017.

[34] S. Bagchi, and S.K. Mitra, "The nonuniform discrete Fourier transform and its applications in signal processing," Springer Science and Business Media, p. 463, 2010.

[35] X. Bai, X. Yang, L.J. Latecki, W. Liu, and Z. Tu, "Learning contextsensitive shape similarity by graph transduction," IEEE Transactions on Pattern Analysis and Machine Intelligence, 32( 5), pp.861-874, 2010. 